\title{
Upper Bounds on the Coarsening Rate of Discrete, Ill-Posed Nonlinear Diffusion Equations
}

\author{
Selim Esedoḡlu and John B. Greer
}

May 23, 2007

\begin{abstract}
We prove a weak upper bound on the coarsening rate of the discrete-in-space version of an ill-posed, nonlinear diffusion equation. The continuum version of the equation violates parabolicity and lacks a complete well-posedness theory. In particular, numerical simulations indicate very sensitive dependence on initial data. Nevertheless, models based on its discrete-in-space version, which we study, are widely used in a number of applications, including population dynamics (chemotactic movement of bacteria), granular flow (formation of shear bands), and computer vision (image denoising and segmentation). Our bounds have implications for all three applications.
\end{abstract}

\section{Introduction.}

We prove a weak upper bound on the coarsening rate of a family of discrete-in-space evolution equations. Although the equations are well-posed, they resemble finite difference approximations of ill-posed nonlinear diffusion equations, leading us to call them discrete ill-posed nonlinear diffusion equations in the title. The equations arise in the study of population dynamics as a class of reinforced random walks on a lattice [14, 24], and in granular flow as a simplified one-dimensional model for the formation of shear bands by anti-plane shear in a granular medium [29]. They also have strong connections to an algorithm introduced by Perona and Malik for image denoising $[10,26]$. Our results apply to the Perona-Malik method in one space dimension.

We study equations that share an interesting phenomenon: a nonlinear instability leads to the formation of spikes (in the case of reinforced random walks) or jump discontinuities (in the granular flow and image processing examples). The spikes and jump discontinuities each have a width of exactly one grid point. After their initial formation, the features do not move laterally, but either grow or shrink until they disappear. The average distance between them establishes a length scale that increases with time at a measurable rate (called the coarsening rate).

For typical applications of these systems, the most relevant questions concern their coarsening behavior; for example, coarsening in the image denoising model represents a gradual simplification of images by merging neighboring regions. In this case, one would like the coarsening to occur as quickly as possible, since this may result in a faster algorithm. In granular flow, the coarsening represents a merging of shear bands, and in population dynamics it represents collective movement to population centers. Our contribution in this paper is to show how recently developed methods for analyzing coarsening rates can be applied to these models to answer questions raised previously [29].

Although the evolution equations appear to be finite difference approximations of PDEs, those PDEs are ill-posed, and the schemes do not converge to them in any obvious way [10]. One could study the PDEs using some type of regularization $[1,2,3,4,6,22]$ or by defining 
an appropriate weak solution [5, 30], but we take a different viewpoint here. We consider applications for which the PDEs serve only as heuristic models for problems that are discrete in nature; for example, digital images and granular media each have a minimum length scale (pixel and grain sizes, respectively) that could be represented by the distance between grid points. In addition, the discrete-in-space equations display all the dynamics essential to the applications - namely, the nonlinear instability and the subsequent coarsening. In fact, it turns out that discreteness plays a crucial role in the coarsening process, as we discuss below. We thus focus our attention on the discrete evolution equations, not the ill-posed PDEs.

Our analysis closely follows a method introduced by Kohn and Otto in [17] for obtaining weak upper bounds on the coarsening rates of energy driven systems. Given the energy $E$ and a quantity $L$ that describes the system length scale, their method requires only a dissipation inequality between $\frac{d L}{d t}$ and $\frac{d E}{d t}$ and an interpolation inequality relating $L$ to $E$. These inequalities are then combined with an ODE argument to prove a time-averaged lower bound on the energy that is conceptually equivalent to an upper bound on the coarsening rate. Kohn and Otto first applied the method to Cahn-Hilliard models for the coarsening of an equal-volume fraction binary mixture [17], and it has more recently been applied to both mean-field [8] and phase-field [9] models of phase separation, in addition to multicomponent phase separation [19], Mullins-Sekerka evolution of a binary mixture in the small volume fraction regime [7], epitaxial growth [18], and thin film droplets [23]. Our discrete setting is different from these previous applications of the method.

In following the arguments of [17] in our discrete setting, we pay close attention to the dependence of estimates on the grid size. Indeed the coarsening rates depend on the uniform grid cell width, $h$, for the finite differences involved. Numerical experiments show that coarsening slows to a halt as $h \rightarrow 0$ [29], so any useful bound on the coarsening rates must account for this dependence. Although our results can be stated in arbitrary space dimensions, for clarity we present them for one and two space dimensions only, and limit our discussion in the first few sections to just one dimension.

The remainder of Section 1 describes the coarsening behavior observed in the discrete-inspace evolution equations (Sections 1.1-1.3), and then explains the overall method of proof and states our main theorem (Section 1.4) for the equations in one dimension. Section 2 is devoted to establishing some basic properties of the dynamics. Section 4 notes how the energy dissipation inequality, one of the ingredients needed in Kohn and Otto's argument, appears in our discrete setting. Section 4 is devoted to establishing the second ingredient, namely the relevant interpolation inequality for our problem. Numerical experiments corroborating our bounds follow in Section 5. Finally, Section 6 shows how to extend the interpolation inequality of Section 4 to two space dimension, allowing us to generalize our main theorem to two dimensions.

\subsection{The Scheme.}

We will work with uniform grids on $[0,1]$ in one space dimension, and on $[0,1]^{2}$ in two

dimensions. In both cases, let $h=\frac{1}{N}$ denote the uniform grid size. The one dimensional scheme that we study is

$$
\frac{d v_{i}}{d t}=D_{h}^{+} D_{h}^{-}\left(R\left(v_{i}\right)\right) \quad i=0, \ldots N-1
$$

where

$$
\begin{aligned}
& D_{h}^{+} v_{i}=\frac{v_{i+1}-v_{i}}{h}, \\
& D_{h}^{-} v_{i}=\frac{v_{i}-v_{i-1}}{h},
\end{aligned}
$$

are forward and backward difference quotients. Periodic boundary conditions will be used, which are common and natural in many applications of these models; thus, when $i=0$ or 
$i=N-1$ we set:

$$
v_{i}=v_{j} \quad j=i \bmod N \quad \text { when } i \neq 0, \ldots N-1 .
$$

Requiring (4) ensures that the dynamics of (1) leave the total mass,

$$
\mu:=\frac{1}{N} \sum_{i} v_{i},
$$

unchanged. This property and all our subsequent results also hold when Neumann boundary conditions are imposed.

System (1) has a non-increasing energy, given by

$$
E(v)=\sum_{i=1}^{N-1} f\left(v_{i}\right) h,
$$

where

$$
f(s)=\int_{0}^{s} R(\xi) d \xi .
$$

For smooth, strictly convex functions $f$, the scheme (1) gives a convergent (as $h \rightarrow 0$ ) finite differences approximation of the parabolic PDE

$$
v_{t}=(R(v))_{x x}=R^{\prime}(v) v_{x x}+R^{\prime \prime}(v) v_{x}^{2}
$$

on the interval $[0,1]$ with periodic boundary conditions. Such systems are well-understood; in this paper we are concerned instead with a special class of non-convex functions $f$ that are convex on some interval $[a, b)$ but concave on $(b, \infty)$. In that case, $R^{\prime}(s)<0$ whenever $s>b$, so that equation (8) becomes backwards parabolic wherever the solution $v$ is large enough.

More precisely, we will make the following assumptions on $R, a$, and $b$ :

$$
\begin{aligned}
& \text { 1. } R: \mathbb{R} \rightarrow \mathbb{R} \text { is smooth, } \\
& \text { 2. } R^{\prime}(s)>0 \text { whenever } s \in(a, b), \\
& \text { 3. } R^{\prime}(s)<0 \text { whenever } s \in(b, \infty) \text {, and } \\
& \text { 4. } R(a) \leq \lim _{s \rightarrow \infty} R(s) \text {. }
\end{aligned}
$$

The switch of sign in $R^{\prime}(s)$ at $s=b$ corresponds to the switch from convexity to concavity in $f$.

In applications, one of the most important examples of such a nonlinearity $R$ is the original choice made by Perona and Malik $[25,26]$ in the context of image processing, namely

$$
R(s)=\frac{s}{1+s^{2}},
$$

a primitive for which is

$$
f(s)=\frac{1}{2} \log \left(1+s^{2}\right) .
$$

In this case $a=0$ and $b=1$. (This nonlinearity also turns out to be relevant in granular flow models, as explained in the next section). We also refer the reader to [20], where coarsening is examined for a model of epitaxial growth that has an energy similar to (11).

Other choices for $R$ that appear in image processing literature and respect the properties listed above include

$$
R(s)=\frac{p s}{\left(1+s^{2}\right)^{\frac{2-p}{2}}} \text { with } p \in(0,1),
$$

a primitive for which is

$$
f(s)=\left(1+s^{2}\right)^{\frac{p}{2}}
$$

Figure 1 shows $f, R$, and $R^{\prime}$ for $f$ given by (11). The bounds we obtain in this paper apply to all these choices; the downside is that our bounds are not sharp enough to distinguish between different choices of nonlinearities. 


\subsection{Applications of (1).}

Using $f$ defined by (11), system (1) describes a reinforced random walk on a lattice and is used to model the movement of biological organisms affected by some external field that is in turn affected by the organisms' presence [14, 24]. Examples of such organisms include ants, which create and follow ant trails, and the bacteria Myxcoccus Xanthus, which glides along a substance produced by other members of the population. Denoting population density by $v$, (1) arises for external fields proportional to the number of entities present.

The dynamics of (8) share many qualitative features with an ill-posed nonlinear PDE that arises in image processing [25, 26] and granular flow [29, 28]:

$$
u_{t}=\left(R\left(u_{x}\right)\right)_{x} .
$$

These similarities should be expected, since (8) may be derived from (13) by letting $v=u_{x}$ and taking a spatial derivative of (13). For functions $f$ such as (11), which have the additional properties of being nonnegative and even with

$$
f(0)=0,
$$

(13) is a one-dimensional restriction of

$$
u_{t}=\nabla \cdot(R(|\nabla u|) \nabla u),
$$

which was introduced by Perona and Malik to denoise digital images represented by the function $u[25,26]$. Intending to smooth noisy regions of the image without blurring edges (object boundaries), they required that $f$, the primitive of $R$, satisfy exactly the conditions discussed above.

Equation (13) has also been studied as a simplified one-dimensional model for the formation of shear bands in a granular medium [29]. In this case, $f$ is defined by

$$
f(s)=\sin \alpha \sin \phi \log \left|\cos \phi+s+\sqrt{1+2 s \cos \phi+s^{2}}\right|+\cos \alpha \sqrt{1+2 s \cos \phi+s^{2}},
$$

with $0<\alpha<\frac{\pi}{2}$ and $0 \leq \phi \leq \pi$. This choice for $f$ has $a=-\infty$ and

$$
b=-\frac{\sin (\alpha-\phi)}{\sin \alpha} .
$$

See [29] for an explanation of the parameters $\alpha$ and $\phi$ in relation to granular flow. Figure 2 includes a comparison of (11) with (15) for $\alpha=\frac{\pi}{4}$ and $\phi=\frac{\pi}{8}$. It is important to note that the behavior of the granular flow nonlinearity (15) at infinity is essentially the same as that of the Perona-Malik nonlinearity (11). Indeed, up to subtracting off a linear term in $s$ (which makes no difference in the resulting flow according to (13)), the function (15) has logarithmic growth at infinity just like (11) does.

Much attention has been devoted to ill-posed equations such as (8) and especially (13). In particular, there is a lot of recent effort that concerns developing an existence theory [16, 15, $30,5,11,12,13]$. Another topic of research has been appropriately regularized versions of the PDEs $[6,3,2,4,1,22]$. But some work regarding the two PDEs has actually focused on discretizations like (1) and the similar discretization of (13),

$$
\frac{d}{d t} u_{i}=D_{h}^{-}\left(R\left(D_{h}^{+} u_{i}\right)\right)
$$

We may derive (1) from (16) by letting

$$
v_{i}=D_{h}^{+} u_{i}
$$

and applying $D_{h}^{+}$to (16). Although the limiting behavior of (1) and (16) as $h \rightarrow 0$ is unclear for non-convex $f$ (see [10] for a particular scaling limit), the schemes have nonetheless been 
studied in relation to each of the above mentioned applications. In image processing, the discretization is actually more important than the intended PDE model, as applications of the model on digital images involve only discretizations of (13). In population dynamics, (1) may be thought of as an example of a reinforced random walk on a lattice. The authors of [28, 29], pointed out that discrete models like (1) are of interest in of granular flow, which is inherently discrete with minimum length scales determined by the grain sizes.

\subsection{Coarsening behavior.}

The one dimensional system (1) under study is a gradient descent for energy (6) with respect to the discrete $H^{-1}$ norm, which can be expressed by duality as

$$
\left\|v_{i}\right\|_{H^{-1}}:=\sup _{\phi_{i}}\left\{\frac{1}{N} \sum_{i=0}^{N}\left(v_{i}-\mu\right) \phi_{i}, \frac{1}{N} \sum_{i=1}^{N}\left[\left(\phi_{i+1}-\phi_{i}\right) N\right]^{2} \leq 1\right\} .
$$

If the initial data $v_{j}(0)$ of $(1)$ satisfies $v_{j}(0) \in(a, b)$ so that it lies completely in the forward parabolic regime, the evolution proceeds as a typical parabolic smoothing. If on the other hand the initial data's mean value $\mu$ satisfies

$$
\mu>b,
$$

then part of the mass of the data always lies in the ill posed regime $(b, \infty)$ due to conservation of mass. In this case, the evolution creates a more interesting behavior: The conserved total mass of the data quickly aggregates into a terrain of spikes. These are locations on the grid where the solution exceeds the parabolicity threshold $b$, i.e. we adopt the following terminology:

$$
\text { There is a spike at the } j \text {-th grid point if } v_{j} \geq b \text {. }
$$

Each spike appears to be supported on a single grid point and does not move; however, the heights of the spikes can change. During subsequent evolution the concavity of $f(s)$ on $(b, \infty)$ encourages accumulation of mass into fewer and bigger spikes. As a result, smaller spikes get absorbed into larger ones until only a single spike containing most of the mass remains (see Figure 3). The growing distance between spikes establishes a system length scale that coarsens.

Possible stationary states for the system can be easily worked out by noting that at equilibrium, one must have $R\left(v_{j}\right)=C$ for all $j$, for some constant $C$. Concentrating on solutions $v_{j}$ with $v_{j} \geq a$ for all $j$, we see from the assumptions on $R$ that this equation can have at most two solutions which we denote $v_{-}$and $v_{+}$, with the assumption that $v_{+}>b>v_{-} \geq a$. Under assumption (19), there is at least one $j$ at which $v_{j}=v_{+}$. Concavity of $f(s)$ at $s=v_{+}$now implies that stable stationary states contain exactly one spike; hence at $(N-1)$ grid points the solution has value $v_{-}$, and at one grid point it has value $v_{+}$. Conservation of mass implies

$$
v_{+}+(N-1) v_{-}=N \mu \text {. }
$$

Together with

$$
R\left(v_{+}\right)=R\left(v_{-}\right)
$$

these two conditions now suffice to solve for $v_{+}$and $v_{-}$given $\mu$. For example, if $f$ is given by (11), then (22) implies

$$
v_{+}=\frac{1}{v_{-}} .
$$

Combining with (21) gives

$$
v_{ \pm}=\frac{N \mu \pm \sqrt{(N \mu)^{2}-4(N-1)}}{2} .
$$


This formula shows in particular that most of the mass is indeed concentrated in the single spike. The gradient descent structure of (1) drives the $v_{i}$ to this energy minimum and may be stopped only by the possible interference of a saddle point corresponding to another stationary state of (1). However, such interference is rare, since the single spike solution is the only stable steady state solution for initial data satisfying (19). A complete study of all steady state solutions of (1), including an investigation of stability, may be found in [21], [24] and [29].

Though the initial aggregation of system mass to spikes occurs rapidly, the evolution slows down as the number of spikes (denoted below by $K$ ) decrease. The authors of [29] used numerical simulations of (1) to measure this coarsening rate and observed that the number of spikes scale like

$$
K \sim\left(\frac{N}{t}\right)^{\frac{1}{3}}
$$

Despite a remarkable correlation with the numerical data (see [29] or Section 5 of this paper), this paper presents the first rigorous result indicating this rate.

The physical meaning of the coarsening process of (1) depends on the particular application. For granular flow, where the energy function given by (15), the coarsening corresponds to a decreasing number of shear bands in the granular medium (see Figure 5). When considering $f$ given by (11), the coarsening represents an aggregation of biological organisms into population centers. For the nonlinearities used in image processing, our analysis applies only to entirely nonnegative data (and may be easily modified for entirely non-positive data). Since we study the signal's discrete derivative, we are examining the coarsening of the terraces produced by the Perona-Malik method along a single edge (see Figure 4). This coarsening corresponds to a simplification of the processed image. At early stages the image is close to the original, perhaps noisy image, while at later stages, fine structures (including noise) disappear and only the larger features remain. An accurate understanding of the coarsening speed of (16) might be used to estimate the computation time needed to process an image up to a desired state of complexity.

\subsection{Our main result and method of proof.}

Once we choose a measurement, $L$, of the system length scale, our analysis requires two inequalities relating $L$ with the system's energy, $E:$ a decay estimate bounding $\frac{d L}{d t}$ by a function of $\frac{d E}{d t}$, and an interpolation inequality that is a one-sided version of

$$
E \sim \frac{1}{L}
$$

We must therefore carefully choose $L$ so that it accurately measures the system length scale while allowing us to prove both required estimates.

Our discussion of the system dynamics suggests choosing $L=\frac{1}{K}$ with $K$ denoting the number of spikes. The interpolation inequality would easily follow for this choice: since $f$ is an increasing function of $v$, on $[a, \infty)$, the $K$ spikes dominate the system energy and

$$
E \sim \frac{K}{N}
$$

On the other hand, a decay estimate for $K$ would be difficult to prove, since it only takes on integer values. Instead we pick

$$
L=\left\|v_{i}\right\|_{H^{-1}}
$$

which is similar to length scales used in [17, 18, 23]. To justify our choice, we provide numerical evidence in Section 5 that $L \sim \frac{1}{K}$.

Having defined $L$, we next state our decay estimate,

$$
|\dot{L}| \leq(-\dot{E})^{\frac{1}{2}}
$$


and our interpolation estimate,

$$
E L \geq \frac{C}{N}
$$

Since (1) is a gradient descent of $E$ with respect to the discrete $H^{-1}$ norm, (26) follows from a slight variation of arguments presented in [17]. The proof of (27) is more interesting. The discrete setting of this problem, which is unlike the setting of other coarsening rate bounds $[8,9,17,18,19,23]$, requires that we pay attention to the dependence of our estimates on the grid size $h=\frac{1}{N}$. Indeed, although our decay estimate relating $E$ with $L$ does not depend on $N$, the interpolation estimate does. We strive for the most favorable dependence we can manage and obtain precisely the one observed in numerical experiments reported in [29].

We present bounds on the coarsening rate in one and two space dimensions. First, we prove the following time averaged lower bound on the energy of the one dimensional scheme (1); the result in 2D is presented in Section 6.

Theorem 1. Suppose $a \leq v_{i}(0)$ for $1 \leq i \leq N$,

$$
b<\mu=\frac{1}{N} \sum_{i} v_{i}
$$

and $v_{i}(0)>b$ for at most $\frac{N}{2}$ values of $i$. Then there exists a universal constant $C<\infty$ such that

$$
\frac{1}{T} \int_{0}^{T} E^{\theta r} L^{-(1-\theta) r} d t \geq C_{I}\left[\left(N^{2} T\right)^{-\frac{1}{3}}\right]^{r}
$$

for $T \geq \frac{\sqrt{3}}{C} N L(0)^{3}$ and for any $0 \leq \theta \leq 1$ and $r>0$ satisfying

$$
r<3, r \theta>1 \text {, and }(1-\theta) r<2 \text {. }
$$

Proof: The decay and interpolation inequalities (26), (27) that will be derived in Sections 3 and 4 , respectively, imply the result once we define the rescaled variables

$$
\tilde{E}=N^{\frac{2}{3}} E \quad \text { and } \quad \tilde{L}=N^{\frac{1}{3}} L
$$

and apply to $\tilde{E}$ and $\tilde{L}$ Lemma 1 that we quote below from [17].

Lemma 1. (From [17]) If $0 \leq \theta \leq 1$ and $r>0$ satisfy (30), then $E L \geq C$ and $(\dot{L})^{2} \leq-\dot{E}$ imply

$$
\frac{1}{T} \int_{0}^{T} E^{\theta r} L^{-(1-\theta) r} d t \geq C T^{-\frac{r}{3}}
$$

for $T \ll L_{0}^{3}$.

Theorem 1 applies to large time behavior of (1), since $K \ll N$ (and is certainly less than $\frac{N}{2}$ ) after a transient initial period where the $v_{i}$ rapidly separate into spikes and background. As discussed in [17], the methods used here will not provide a lower bound on the coarsening rate of (1). Proving a suitable lower bound would likely be more difficult, since the dynamics of (1) may be slowed by a variety of factors, including the interference of saddle points of the energy; hence there is in fact no lower bound in the naive sense. On the other hand, a system can not coarsen any faster than its energy landscape allows.

\section{Preliminaries.}

We consider only initial data satisfying $v_{i}(0) \geq a$. The following lemma shows that the $v_{i}$ retain this lower bound at all later times. 
Lemma 2. Suppose $v_{i}$ solves (1) for $t \in[0, T)$ and satisfies

$$
v_{i}(0) \geq a
$$

for $i=0, \ldots, N-1$. Then a solution $v_{i}$ exists for all later times and

$$
v_{i}(t) \geq a
$$

for $i=0, \ldots, N-1$ and all $t \geq 0$.

Proof. Our assumptions on the function $R$ (Section 1.1) imply it is globally Lipschitz on $[a-1, \infty)$. Standard ODE theory implies the existence of a $T>0$ so that solutions to the following ODE systems can be found on $[0, T]$ :

$$
\begin{aligned}
\frac{d v^{\varepsilon}}{d t} & ==\frac{R\left(v_{i+1}^{\varepsilon}(t)\right)-2 R\left(v_{i}^{\varepsilon}(t)\right)+R\left(v_{i-1}^{\varepsilon}(t)\right)}{h^{2}}+\varepsilon \\
v_{i}^{\varepsilon}(0) & =v_{i}(0)
\end{aligned}
$$

under the hypothesis on $v_{i}(0)$. Also, $v_{i}^{\varepsilon} \rightarrow v_{i}$ uniformly on $[0, T]$ as $\varepsilon \rightarrow 0$, provided that $T>0$ is small enough. We show that for small enough $\varepsilon>0, v_{i}^{\varepsilon}(t)>a-\varepsilon$ for all $i$ and $t \in[0, T]$. Fix an $\varepsilon>0$ and assume not. Let $t_{*} \in(0, T]$ be the first time there is a $j$ such that $v_{j}^{\varepsilon}\left(t_{*}\right)=a-\varepsilon$. The whole point is:

$$
R(s) \geq a \text { for all } s \geq a
$$

according to the assumptions (9) on the function $R$. Hence, we must have

$$
\frac{d}{d t} v_{j}^{\varepsilon}=\frac{1}{h^{2}}\left(R\left(v_{j+1}^{\varepsilon}\right)+R\left(v_{j-1}^{\varepsilon}\right)-2 R(a)\right)+\varepsilon \geq \varepsilon>0
$$

for all $t \in\left[0, t_{*}\right]$ close enough to $t_{*}$. This is a contradiction since $a-\varepsilon$ is the strict minimum of $v_{j}(t)$ on $t \in\left[0, t_{*}\right]$. Taking $\varepsilon \rightarrow 0$ now proves

$$
v_{i}(t) \geq a
$$

for all $t \in[0, T]$. That in return implies the solution extends to all $t \geq 0$ and remains above $a$ for all time.

We next separate the $v_{i}$ into spikes and background. Recall that we call $v_{i}$ a spike if $v_{i}>b$, where $b$ is the parabolicity threshold discussed previously. By selecting $b$ as the dividing value, we ensure that the number of spikes does not increase:

Lemma 3. If

$$
v_{i}\left(t_{0}\right) \leq b
$$

for some $i$ and some $t_{0} \geq 0$, then

$$
v_{i}(t) \leq b
$$

for all $t \geq t_{0}$.

Proof. The same argument from previous Lemma 2 can be applied, but now the essential fact is that $R(s)$ achieves its maximum at $s=b$. Indeed, we can consider the system

$$
\frac{d}{d t} v_{i}^{\varepsilon}=\frac{R\left(v_{i+1}(t)\right)-2 R\left(v_{i}(t)\right)+R\left(v_{i-1}(t)\right)}{h^{2}}-\varepsilon
$$

Assuming that the conclusion is false for some grid point $j$, one sees that at the first time $t_{*}$ at which $v_{j}^{\varepsilon}\left(t_{*}\right)=b$, it has to be the case that

$$
\left.\frac{d}{d t} v_{j}^{\varepsilon}=\frac{1}{h^{2}}\left(R\left(v_{j+1}^{\varepsilon}\right)+R\left(v_{j-1}^{\varepsilon}\right)-2 R(b)\right)\right)-\varepsilon<0
$$

which leads to a contradiction as before. 


\section{Decay relation.}

\section{Lemma 4.}

$$
|\dot{L}| \leq(-\dot{E})^{\frac{1}{2}}
$$

Proof. Our selected length scale (18) allows a simple application of the proof of Lemma 2 in [17] to this discrete setting. We provide the details only for the reader's benefit. From (6), we have

$$
-\dot{E}=-\sum_{i=0}^{N-1} R\left(v_{i}\right) \dot{v}_{i} h=-\sum_{i=0}^{N-1} R\left(v_{i}\right) D_{h}^{+} D_{h}^{-}\left(R\left(v_{i}\right)\right) h .
$$

Summing by parts and using the boundary condition (4) gives

$$
-\dot{E}=\sum_{i=0}^{N-1}\left[D_{h}^{-}\left(R\left(v_{i}\right)\right)\right]^{2} h
$$

Pick $t_{1}, t_{2}$ with $0 \leq t_{1}<t_{2}$ and let $\xi$ be the optimal test function in the definition of $L\left(t_{2}\right)$ (see (18) and (25)) so that

$$
L\left(t_{2}\right)=\left\|v\left(t_{2}\right)\right\|_{H^{-1}}=\sum_{i=0}^{N-1}\left(v_{i}\left(t_{2}\right)-\mu\right) \xi_{i} h
$$

and

$$
\sum_{i=0}^{N-1}\left|D_{h}^{+} \xi_{i}\right|^{2} h \leq 1
$$

Then we have

$$
\begin{aligned}
L\left(t_{2}\right)-L\left(t_{1}\right) & \leq \sum_{i=0}^{N-1}\left(v_{i}\left(t_{2}\right)-v_{i}\left(t_{1}\right)\right) \xi_{i} h \\
& =\int_{t_{1}}^{t_{2}} \sum_{i=0}^{N-1} \dot{v}_{i} \xi_{i} h d t \\
& =\int_{t_{1}}^{t_{2}} \sum_{i=0}^{N-1} D_{h}^{+} D_{h}^{-}\left(R\left(v_{i}\right)\right) \xi_{i} h d t \\
& =-\int_{t_{1}}^{t_{2}} \sum_{i=0}^{N-1} D_{h}^{-}\left(R\left(v_{i}\right)\right) D_{h}^{-} \xi_{i} h d t \\
& \leq \int_{t_{1}}^{t_{2}}\left(\sum_{i=0}^{N-1}\left|D_{h}^{-}\left(R\left(v_{i}\right)\right)\right|^{2} h\right)^{\frac{1}{2}} d t
\end{aligned}
$$

Repeating the above with $\xi_{i}^{\prime}$ optimal in the definition of $L\left(t_{1}\right)$ gives

$$
\left|L\left(t_{2}\right)-L\left(t_{1}\right)\right| \leq \int_{t_{1}}^{t_{2}}\left(\sum_{i=0}^{N-1}\left|D_{h}^{-}\left(R\left(v_{i}\right)\right)\right|^{2} h\right)^{\frac{1}{2}} d t .
$$

Combining (33) with (32) shows that $L$ is an absolutely continuous function of $t$ satisfying (26).

\section{Interpolation inequality}

In this section, we establish the second ingredient necessary for applying Kohn and Otto's technique to our problem: an interpolation inequality between the system's energy (6) and 
length scale (25). The following lemma establishes the required inequality in one space dimension; we present the two dimensional version in Section 6.1.

Recall that we work on the unit interval $[0,1]$ divided into a uniform grid with $N+1$ points $0=x_{0}<x_{1}<\ldots<x_{N}=1$, where $x_{i}=\frac{i}{N}$. Let $\left\{v_{i}\right\}$ be a function defined on the grid, satisfying the boundary condition $v_{N}=v_{0}$. As before, $K$ denotes the number of spikes in $v$, i.e. the number of indices $i \in\{0, \ldots, N-1\}$ where $v_{i} \geq b$.

Lemma 5. Let $L$ be defined by (25) and (18), and $E$ by (6). Assume that $K \leq \frac{N}{2}$. Then

$$
E L \geq \frac{C}{N}
$$

for some $C>0$ that depends only on $\mu$ and $b$.

\section{Proof:}

Since $f$ is a non-negative function with $f(s) \geq f(b)$ for all $s \geq b$, we have the immediate lower bound

$$
E \geq \frac{K}{N} f(b),
$$

and we only need to show that $L \geq \frac{C}{K}$ for some constant $C$ depending only on $\mu$ and $b$. It is convenient to establish this using the characterization of the $H^{-1}$-norm given in (18). In particular, we construct a "test function" $\left\{\phi_{i}\right\}$ to get from (18) a lower bound on $\|v\|_{H^{-1}}$. To that end, define the integer $\ell$ as

$$
\ell=\left\lfloor\frac{N}{2 K}\right\rfloor \text {. }
$$

Note that $\ell \geq 1$ since $K \leq \frac{N}{2}$ by hypothesis. It is easy to see that, for the same reason,

$$
\ell \geq \frac{N}{4 K}
$$

We now partition the grid into $2 K$ adjacent, disjoint intervals $I_{j}$, each containing the indices of $\ell$ consecutive grid points, i.e.

$$
I_{j}=\{i: \ell j \leq i<\ell(j+1)\} .
$$

There are exactly $2 K$ such subintervals, $I_{0}, \ldots, I_{2 K-1}$, on the grid. Since there are only $K$ spikes, we see that at least $K$ of the subintervals must contain no spikes. Let

$$
S=\left\{j: I_{j} \text { contains no spikes. }\right\}
$$

so that

$$
\# S \geq K
$$

We now define the test function $\left\{\phi_{i}\right\}$ on the grid as follows: On a subinterval that contains no spikes, we let $-\phi$ be the distance function to the boundary of the subinterval; specifically,

$$
\phi_{i}=-\frac{1}{N} \min \left\{\left(i-\ell j+\frac{1}{2}\right),\left(\ell(j+1)+\frac{1}{2}-i\right)\right\} \text { if } i \in I_{j} \text { for some } j \in S .
$$

Everywhere else, we let $\phi \equiv 0$, i.e.

$$
\phi_{i}=0 \text { if } i \notin \bigcup_{j \in S} I_{j}
$$

Then, first of all, according to (38) and (39), $\left|D^{+} \phi_{i}\right| \leq 1$ for all $i$, so that

$$
\|\phi\|_{H^{1}} \leq 1
$$


Second, if $I$ is a subinterval containing no spikes, then

$$
\frac{1}{N} \sum_{i \in I}\left(-\phi_{i}\right) \geq \frac{\ell^{2}}{4 N^{2}}
$$

which is also an easy consequence of our definition (38) above.

We can now use the test function $\phi$ in the definition (18) of the $H^{-1}$ norm. First, note that due to assumption (28), we have

$$
v_{i}<b<\mu \text { whenever } i \in \bigcup_{j \in S} I_{j}
$$

Then,

$$
\begin{aligned}
L:=\|v\|_{H^{-1}} & \geq \frac{1}{N} \sum_{i=0}^{N-1} \phi_{i}\left(v_{i}-\mu\right) & & \text { (by (18) and (40)) } \\
& =\frac{1}{N} \sum_{j \in S} \sum_{i \in I_{j}} \phi_{i}\left(v_{i}-\mu\right) \geq \frac{1}{N} \sum_{j \in S}(\mu-b) \sum_{i \in I_{j}}\left(-\phi_{i}\right) & & \text { (by (39) and (42)) } \\
& \geq(\mu-b) \sum_{j \in S} \frac{\ell^{2}}{4 N^{2}} & & \text { (by (38) and (41)) } \\
& \geq(\mu-b) K\left(\frac{1}{4 N^{2}}\right)\left(\frac{N^{2}}{16 K^{2}}\right)=(\mu-b) \frac{1}{64 K} & & \text { (by (37) and (36)). }
\end{aligned}
$$

Combined with (34), this establishes the claim with $C=\frac{(\mu-b)}{64}$.

\section{Numerical evidence.}

We next demonstrate the actual coarsening rate of (1), by discretizing in time and solving numerically. In particular, we show a dependence of the coarsening rate on $N$, as our upper bound indicates. We use $f$ defined by (11) in all examples and remark that similar results are discussed in [29] for $f$ given by (15).

Let

$$
F\left(v_{i}^{n}\right)=\frac{R\left(v_{i+1}^{n}\right)-2 R\left(v_{i}^{n}\right)+R\left(v_{i-1}^{n}\right)}{h^{2}} .
$$

We remind the reader that $h=\frac{1}{N}$ and note that we intermittently use $h$ instead of $N$, since that notation is likely more familiar to those having experience with numerical PDEs. We first consider the forward Euler method,

$$
\frac{v_{i}^{n+1}-v_{i}^{n}}{\tau}=F\left(v_{i}^{n}\right)
$$

To demonstrate the coarsening rate of (48), we use a perturbation of $v_{i}=3$ for initial data:

$$
v_{i}(0)=3+10^{-6} \sin \left(\frac{\pi i}{N}\right) .
$$

Notice that in a system with $N$ grid points, $K=N$ for (49), since $b=1$ for $f$ given by (11). Figure 6 shows a plot $\frac{K}{N}$ versus $N^{2} t$ for the solution of (48)with $N=200,300, \ldots, 1400,1500$. After a transient initial period, the coarsening strongly corresponds with the predicted power law $\left(N^{2} t\right)^{-\frac{1}{3}}$. Our analysis does not include the early time dynamics, since our results only hold for the later times when $K \leq \frac{N}{2}$. Figure 7 shows a plot of $L N$ versus $N^{2} t$. Its correspondence with with the power law $\left(N^{2} t\right)^{\frac{1}{3}}$ supports our claim that $L \sim \frac{1}{K}$. The quantity $L$ as given by the $H^{-1}$ norm (18) was computed using the following equivalent characterization:

$$
\|v\|_{H^{-1}}^{2}=\frac{1}{N} \sum_{j}\left(D_{h}^{+} w_{j}\right)^{2} \text { where } D_{h}^{+} D_{h}^{-} w=v \text { with periodic b.c. }
$$


Since the explicit scheme (48) restricts time steps to be $O\left(h^{2}\right)$, one might naturally turn to implicit schemes with weaker time step requirements. Surprisingly, the error caused by taking relatively larger time steps in such schemes significantly slows the coarsening process. To demonstrate this phenomenon, we focus on the implicit midpoint method,

$$
\frac{v_{i}^{n+1}-v_{i}^{n}}{\tau}=F\left(\frac{v_{i}^{n}+v_{i}^{n+1}}{2}\right),
$$

which has less stringent time step requirements than (48) and is typically more accurate than the backward Euler method. Figure 8 shows results for (51) when time steps are not taken larger than $10 h^{2}$. The coarsening occurs significantly slower, especially for large $\mathrm{N}$ (small h). We use Newton's method to solve (51) at each time step, using explicit time stepping to provide an initial guess for the Newton iterations. To ensure rapid convergence of the iterations, we use an adaptive time step - reducing the time step when many iterations are required, and increasing when only 0-5 iterations are required. We use the Sherman-Morrison formula [27] and the tridiagonal matrix solver in LAPACK to solve the linear systems for each Newton iteration. Typically smaller time steps are required only at the beginning stages of evolution.

We further demonstrate the slow-down due to larger time steps by fixing $N=200$ and solving (51) to a fixed time $t_{*}$ for a range of maximal time steps. Figure 9 shows a plot of $K$ at $t_{*}=10$ as a function of the maximum time step used in computation. These results should be of interest to those in the image processing community - in image processing applications, the length scale is more important than the actual time, which is in fact artificial. In particular, these results suggest that while implicit time stepping may remove time step restrictions, little real gain in processing speed might be made.

\section{Two dimensions.}

We easily generalize (1) to two dimensions by considering a system $v_{i, j}, i, j=0, \ldots, N-1$ satisfying

$$
\dot{v}_{i, j}=D_{1, h}^{+} D_{1, h}^{-}\left(R\left(v_{i, j}\right)\right)+D_{2, h}^{+} D_{2, h}^{-}\left(R\left(v_{i, j}\right)\right)
$$

with the rules

$$
v_{i, j}=v_{i \bmod N, j \bmod N} \text { for } i, j \neq 0, \ldots, N-1
$$

and

$$
v_{i, j} \geq a \text {. }
$$

Here, $D_{i, h}^{+}$and $D_{i, h}^{-}$are the forward and backward difference quotient operators, respectively, in the $i$-th coordinate direction. This system is a standard centered differencing discretization of

$$
v_{t}=\Delta(R(v))
$$

with periodic boundary conditions. Although (52) has no relation to the Perona-Malik method for image denoising or to shearing in granular materials, it is a natural generalization of the reinforced random walk model to a two dimensional lattice, and has been studied in [14, 24]. The behavior of (52) is very similar to (1), with mass aggregating in spikes that coarsen with time. System (52) has

$$
E\left(v_{i, j}\right)=\sum_{i, j=0}^{N-1} f\left(v_{i, j}\right) h^{2}
$$

for an energy. Condition (53) ensures that

$$
\mu:=\frac{1}{N^{2}} \sum_{i, j=1}^{N} v_{i, j}
$$


does not change.

We generalize the definition of $L$ to this two dimensional lattice:

$L\left(v_{i, j}\right)=\left\|v_{i, j}\right\|_{H^{-1}}:=\sup _{\phi_{i, j}}\left\{\frac{1}{N^{2}} \sum_{i, j=0}^{N-1}\left(v_{i, j}-\mu\right) \phi_{i, j}, \sum_{i, j=0}^{N-1}\left[\left(\phi_{i+1, j}-\phi_{i, j}\right)^{2}+\left(\phi_{i, j+1}-\phi_{i, j}\right)^{2}\right] \leq 1\right\}$.

In this case, we expect $L \sim \frac{1}{\sqrt{K}}$ where $K$ is again the number of $v_{i, j}>b$. Lemmas 2-4 are all easily seen to hold also for (52); however the interpolation inequality is different as it depends on dimension.

\subsection{Interpolation inequality}

Lemma 6. Let $L$ be defined by (58) and $E$ by (56). Assume that $K \leq \frac{N}{2}$. Then,

$$
E L^{2} \geq \frac{C}{N^{2}}
$$

for some constant $C>0$ that depends only on $\mu$ and $b$.

\section{Proof.}

We proceed in analogy with the one dimensional case covered in Lemma 5. The setting is now the domain $[0,1]^{2}$, discretized by the uniform grid $\left(x_{i}, y_{j}\right)$ where $x_{i}=\frac{i}{N}$ and $y_{j}=\frac{j}{N}$. This time, we have the immediate bound

$$
E \geq \frac{K}{N^{2}} f(b)
$$

on the energy. Hence, it will be enough to show that $L \geq \frac{C}{\sqrt{K}}$ for some $C$. To that end, we will partition the grid into disjoint cubes of side length

$$
\ell=\left\lfloor\frac{N}{\sqrt{2 K}}\right\rfloor .
$$

Then, $\ell \geq \frac{N}{2 \sqrt{2 K}}$. The cubes $Q_{m, n}$ are:

$$
Q_{m, n}=\{(i, j): \ell m \leq i<\ell(m+1) \text { and } \ell n \leq j<\ell(n+1)\} .
$$

There are $2 K$ such cubes completely contained on the grid. Since there are at most $K$ spikes, at least $K$ of these cubes contain no spikes at all. Let

$$
S=\left\{(m, n): Q_{m, n} \text { contains no spikes } .\right\} .
$$

As before, we define a "test function" $\left\{\phi_{i, j}\right\}$ on the grid, as follows:

1. If $(i, j) \in Q_{m, n}$ for some $(m, n) \in S$, then

$$
\begin{aligned}
\phi_{i, j} & =-\operatorname{dist}_{\partial Q_{m, n}}(i, j) \\
& =\min \left\{\left(i-\ell m+\frac{1}{2}\right),\left(\ell(m+1)+\frac{1}{2}-i\right),\left(j-\ell n+\frac{1}{2}\right),\left(\ell(n+1)+\frac{1}{2}-j\right)\right\}
\end{aligned}
$$

2. Otherwise, $\phi_{i, j}=0$.

It follows that $\|\phi\|_{H^{-1}} \leq 1$. Moreover, if $(m, n) \in S$, then

$$
\frac{1}{N^{2}} \sum_{(i, j) \in Q_{m, n}}\left(-\phi_{i, j}\right) \geq C \frac{\ell^{3}}{N^{3}}
$$


for some constant $C$. Using $\phi_{i, j}$ in (58) to bound $L$ from below, we get:

$$
\begin{aligned}
L:=\|v\|_{H^{-1}} & \geq \frac{1}{N^{2}} \sum_{i, j} \phi_{i, j}\left(v_{i, j}-\mu\right) \\
& =\frac{1}{N^{2}} \sum_{(m, n) \in S} \sum_{(i, j) \in Q_{m, n}} \phi_{i, j}\left(v_{i, j}-\mu\right) \geq \frac{1}{N^{2}} \sum_{(m, n) \in S}(\mu-b) \sum_{(i, j) \in Q_{m, n}}\left(-\phi_{i, j}\right) \\
& \geq(\mu-b) \sum_{(m, n) \in S} C \frac{\ell^{3}}{N^{3}} \\
& \geq C(\mu-b) K\left(\frac{N^{3}}{K \sqrt{K}}\right)\left(\frac{1}{N^{3}}\right)=\frac{C(\mu-b)}{\sqrt{K}} .
\end{aligned}
$$

Combined with (60), this establishes the claim.

\subsection{Final result in two dimensions.}

Theorem 2. Suppose $a \leq v_{i, j}(0)$ for $1 \leq i, j \leq N$,

$$
b<\mu=\frac{1}{N^{2}} \sum_{i, j=1}^{N} v_{i, j}
$$

and $v_{i, j}(0)>$ for at most $\frac{N^{2}}{2}$ ordered pairs $(i, j)$. Then for each $\sigma \in(1,2]$, there exist universal constants $C_{1}(\sigma), C_{2}(\sigma)<\infty$ such that for all $0<T_{0} \leq T$,

$$
\frac{1}{T-T_{0}} \int_{T_{0}}^{T} E^{\sigma} d t \geq C_{1}(\sigma)\left[\left(N^{2}\left(T-T_{0}\right)\right)^{-\frac{1}{2}}\right]^{\sigma}
$$

for $\left(T-T_{0}\right) \geq C_{2}(\sigma) N^{2} L\left(T_{0}\right)^{4}$. If $1<\sigma<2$, (68) also holds for $T_{0}=0$.

Proof. The proof may be obtained by setting $\tilde{E}=N E$ and $\tilde{L}=N^{\frac{1}{2}} L$, and then using inequalities (26) and (59) to apply Lemma 4.2 in [23] (with $\alpha=2$ in the statement of that lemma) to $\tilde{E}$ and $\tilde{L}$.

\subsection{Numerical evidence for the $2 \mathrm{D}$ case.}

We use Forward Euler time stepping (48) to solve (52). Although we observed a stability restriction on the explicit scheme of about $\tau \leq \frac{h^{2}}{4}$, standard implicit schemes provide little speed-up, due to the computation required by repeated Newton iterations and the slow down of coarsening caused by large time steps (see Section 5).

We evolve an $N \times N$ system with $N=2^{n} \times 10$ for $1 \leq n \leq 6$. Figure 10 shows $\frac{K}{N^{2}}$ versus $N t$ compared with the power law $\left(N^{2} t\right)^{-\frac{1}{2}}$. Each simulation has the same initial condition,

$$
v_{i, j}(0)=3+10^{-6} \sin \left(\frac{i \pi}{N}\right) \sin \left(\frac{j \pi}{N}\right) .
$$

The results match our proved bound (68).

\section{Discussion.}

Our numerical experiments indicate that not only are the upper bounds we establish attained, but they are in fact generically observed for energy densities with logarithmic growth at infinity, such as (11) that appear in many applications. Our bounds certainly hold for energy densities that have faster (e.g. power law) growth at infinity; the hypothesis of our claims 
clearly allow them. However, numerical experiments indicate that the bounds are not optimal in these cases. It would be interesting to see how the bounds could be improved for these energies, since this would help clarify the role of different nonlinearities in models such as Perona-Malik.

We studied evolution equations that are continuous in time, but those computing these systems (particularly in the image processing community) also care about discrete-in-time versions. The two should be closely related for sufficiently small time steps, but anyone interested in reducing computation time would prefer taking the largest time steps possible. In Section 5 we discussed a slow-down of coarsening caused by taking larger time steps in numerical simulations. This leads one to ask whether a bound on the coarsening rate can be found in terms of the number of time steps in discrete-in-time systems, possibly indicating the amount of computation needed to denoise images with the Perona-Malik method.

Our work focuses on problems best described by discrete-in-space evolution equations, but it would be interesting to see whether our results could be extended to the notions of solution developed for the related ill-posed PDEs in recent literature (e.g. [5, 30]). One might also apply these methods to regularized versions of the PDEs, such as those analyzed in $[1,2,3,4,6,22]$. In this case, we might expect to find that the coarsening rate depends on the regularization parameter just as the coarsening rate depends on grid cell size for the discrete-in-space equations.

Acknowledgments. We thank Robert V. Kohn for recommending this problem, and for his advice and encouragement throughout the paper's development. We also thank Felix Otto and Dejan Slepčev for helpful comments. 


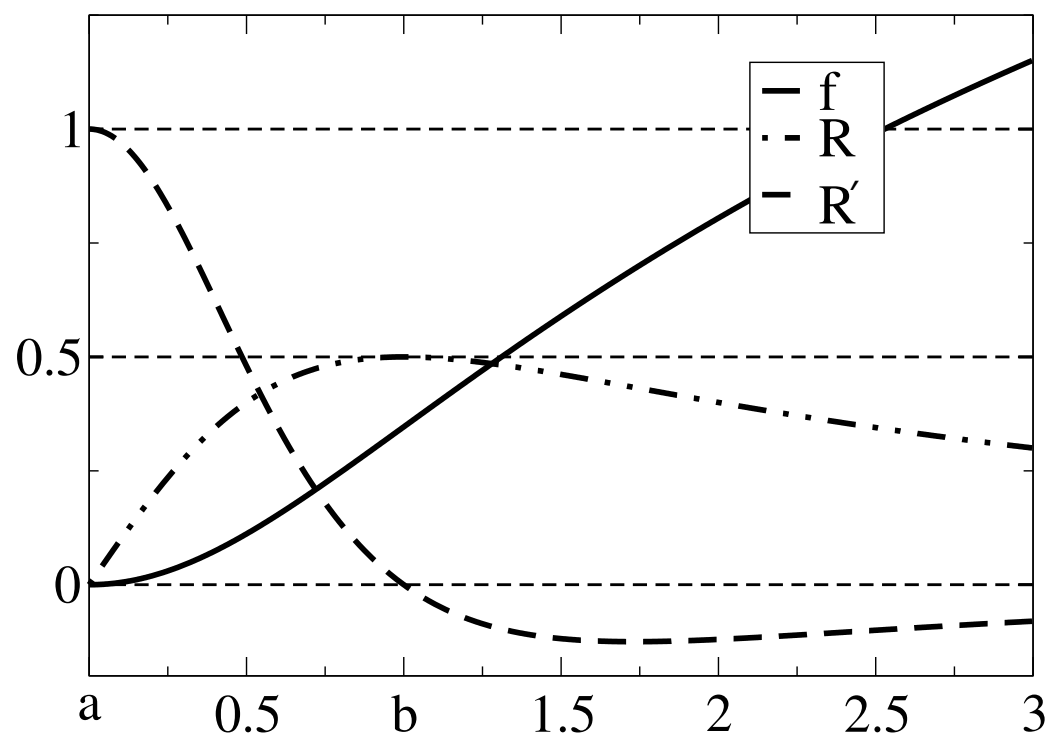

Figure 1: The functions $f, R$, and $R^{\prime}$ for $f(s)=\frac{1}{2} \log \left(1+s^{2}\right)$.
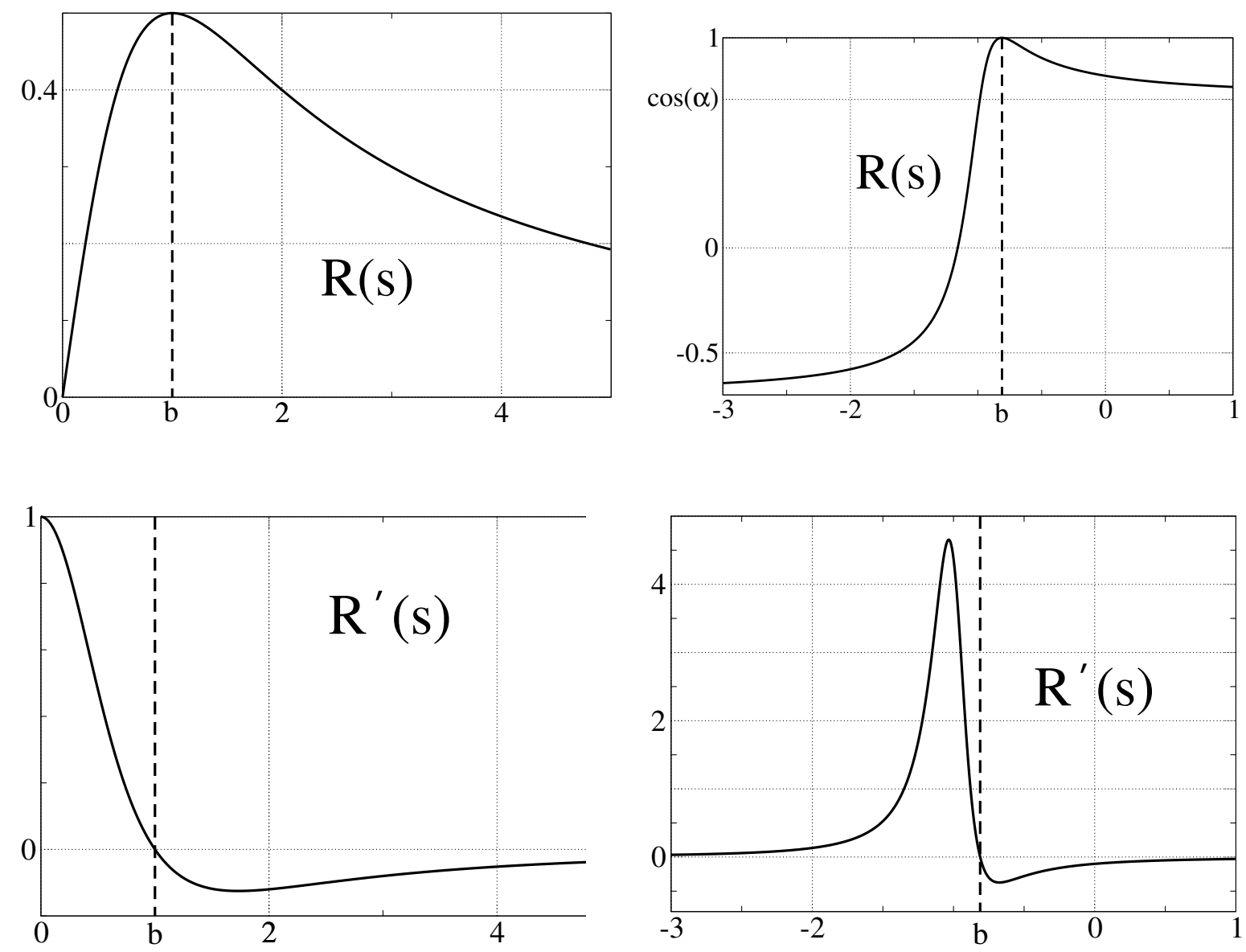

Figure 2: Comparison of $R$ and $R^{\prime}$ when $f$ is defined by (11) (left) and (15) (right). 

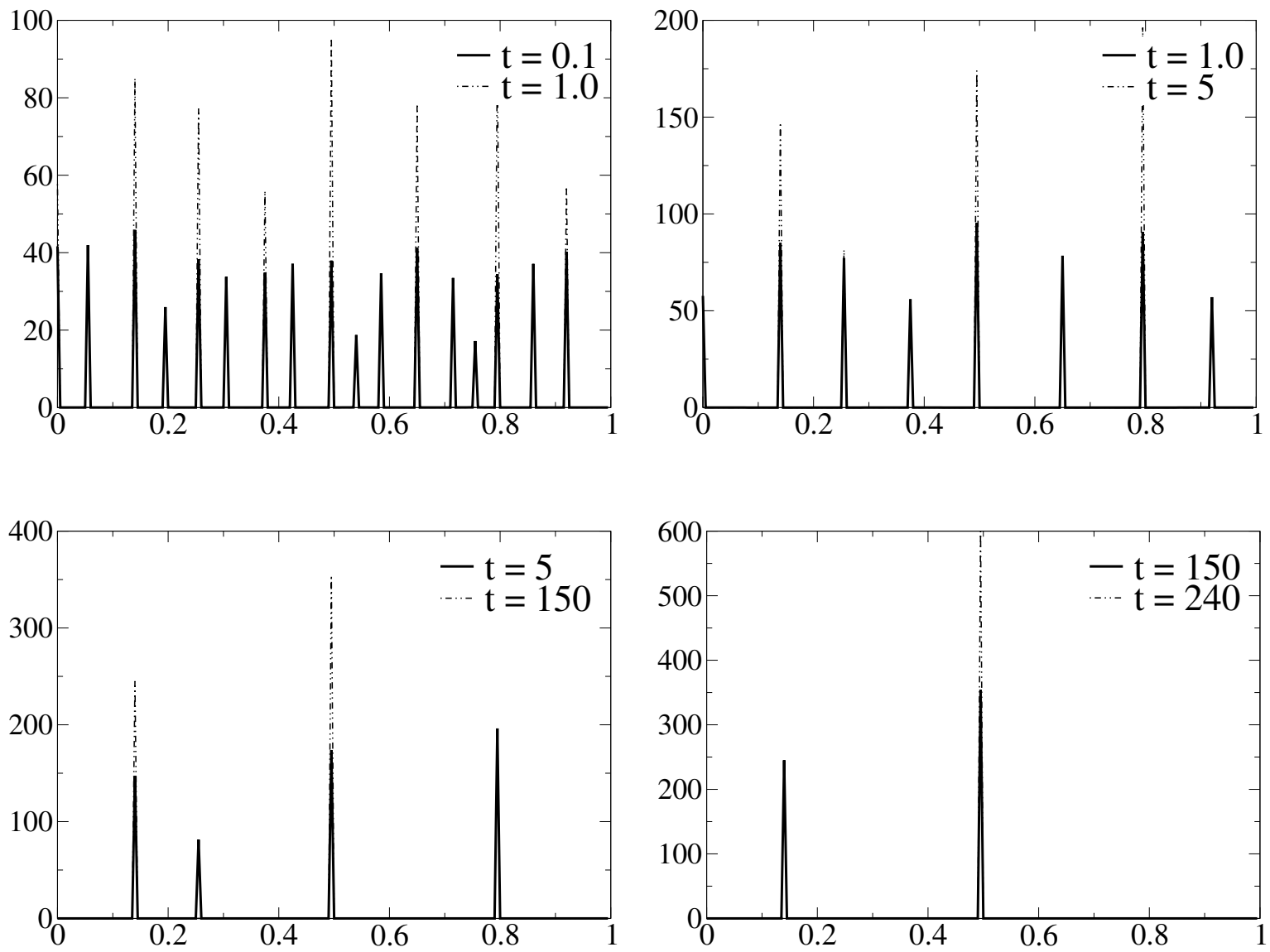

Figure 3: The dynamics of (1) for $N=200$ and $v_{i}(0)=3+\eta_{i}$ with a random perturbation $\left|\eta_{i}\right| \leq$ $10^{-5}$. The $v_{i}$ quickly aggregate to spikes that decrease in number until a single spike contains most of the mass. 


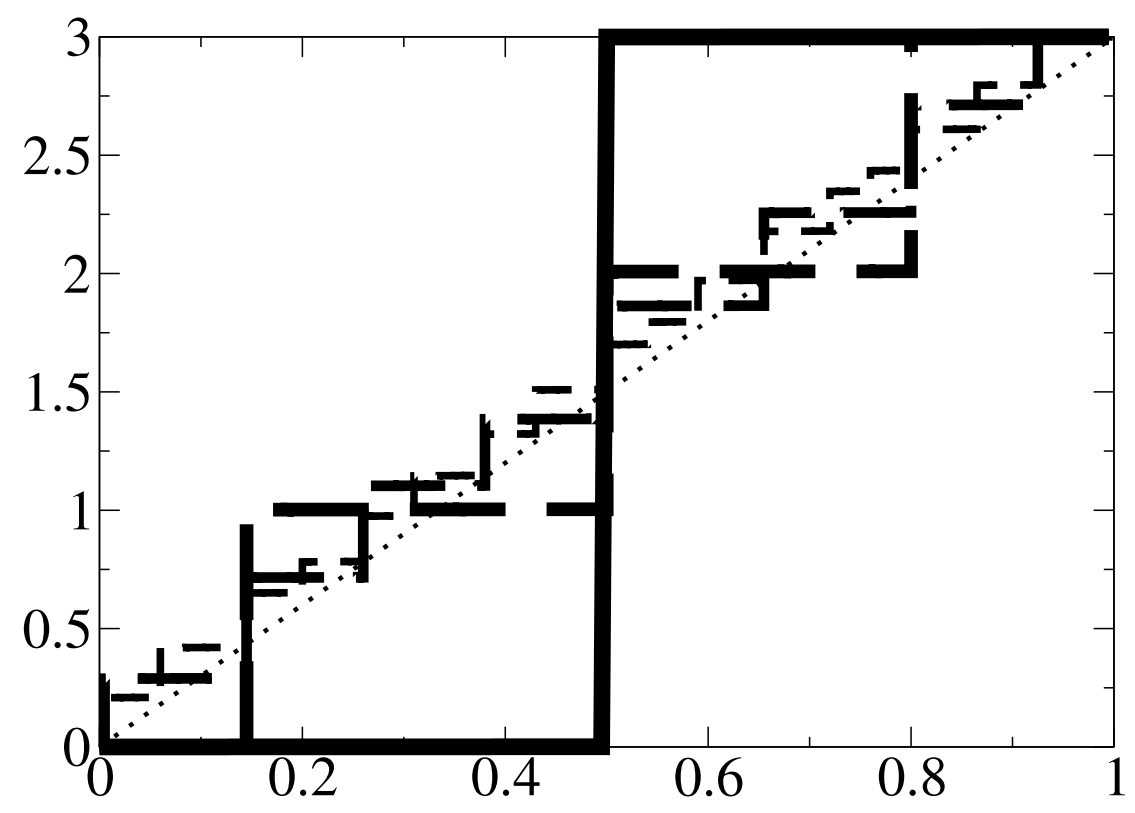

Figure 4: Perona-Malik equation. The dynamics of (16) for $N=200$ and $f$ given by (11). Thicker lines denote later stages of the evolution.

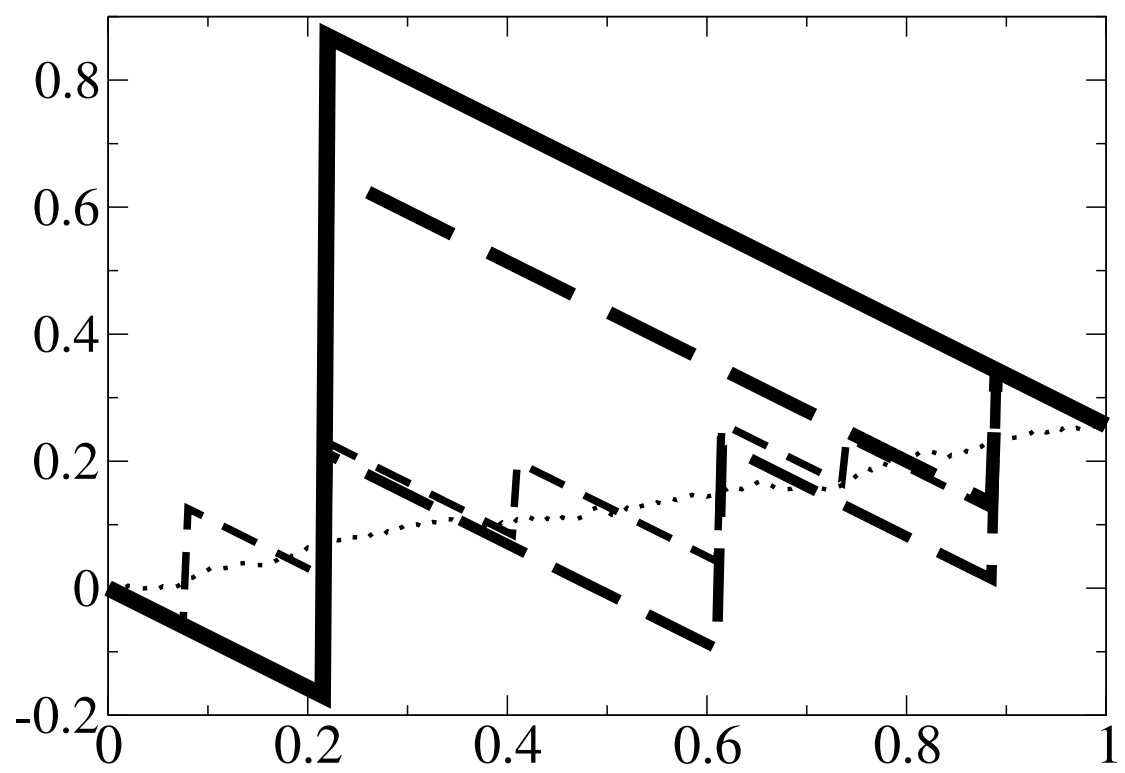

Figure 5: Shear bands in granular flow. The dynamics of (16) for $N=200$ and $f$ given by (15). Thicker lines denote later stages of the evolution. 


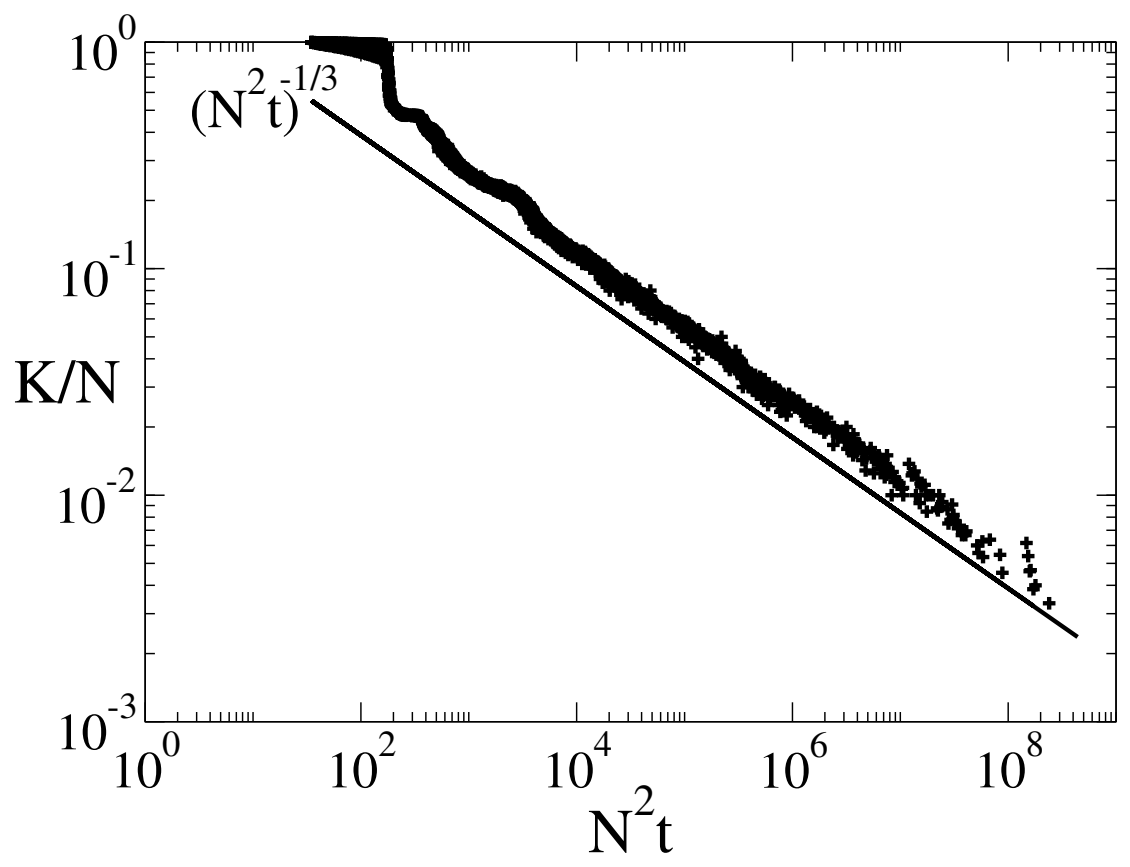

Figure 6: After an initial transient period, the jump density, $\frac{K}{N}$ corresponds closely with the proved coarsening bound (29).

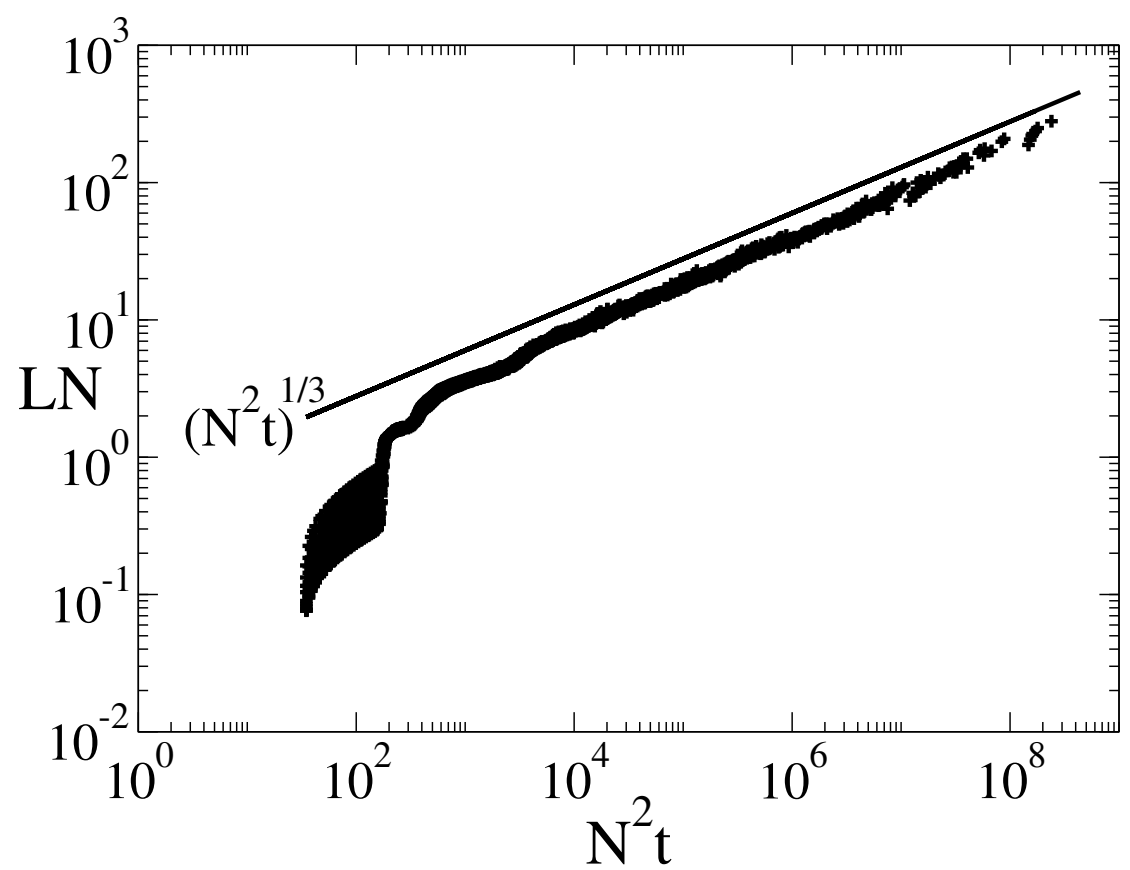

Figure 7: The length scale behaves like $L \sim \frac{1}{K}$, supporting our claim that (25) defines a valid measure of length scale. 


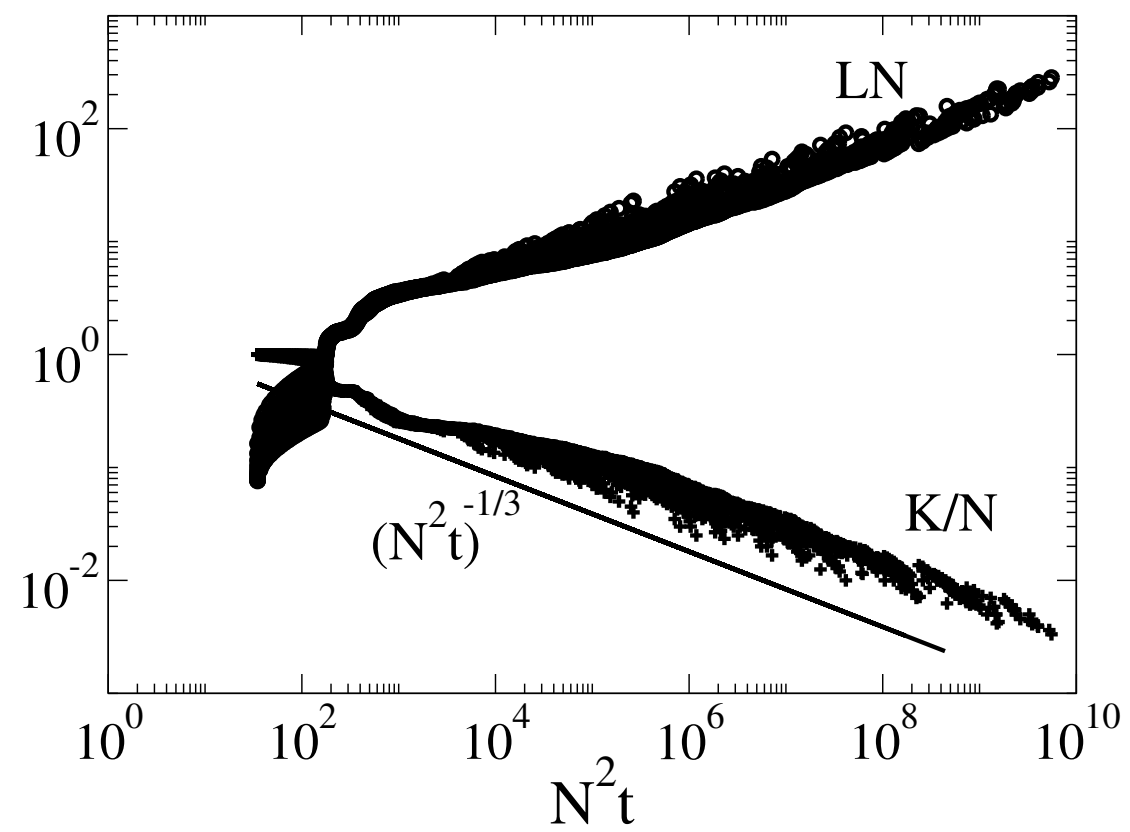

Figure 8: Evolving length scale (top) and jump density (bottom) for the implicit scheme (51). Although implicit schemes have no time step restriction for stability, the coarsening rate badly reflects the error caused by larger time steps. 


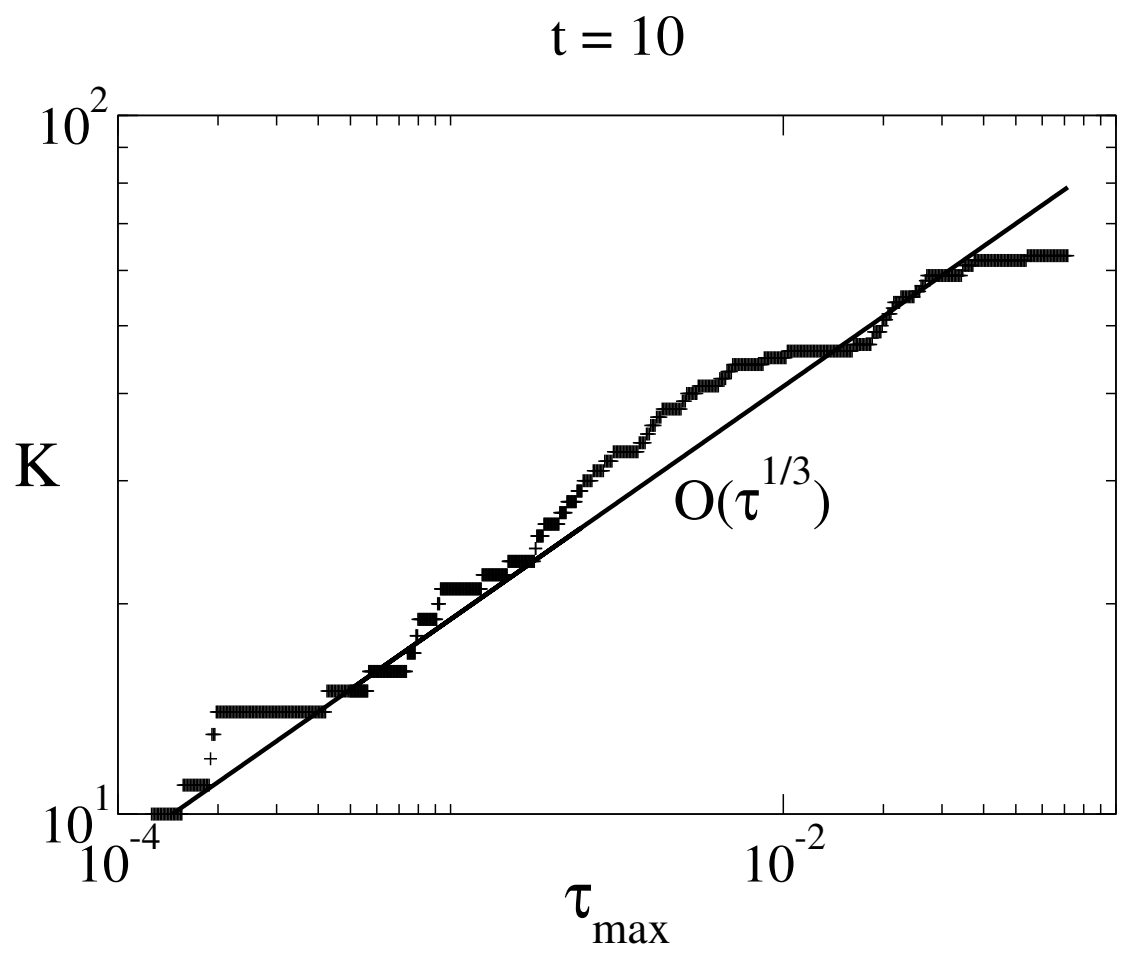

Figure 9: Coarsening slow-down. Although implicit schemes have no time step requirements for stability, errors due to large time steps have a significant effect on the coarsening rate. Using the initial data given by (49), we set $N=200$ and plot $K$ at $t=10$ as a function of the maximal time step used in (51) (this is the time step used except at the earliest stages of evolution, where $\tau$ may be smaller to ensure convergence of the Newton iterations). 


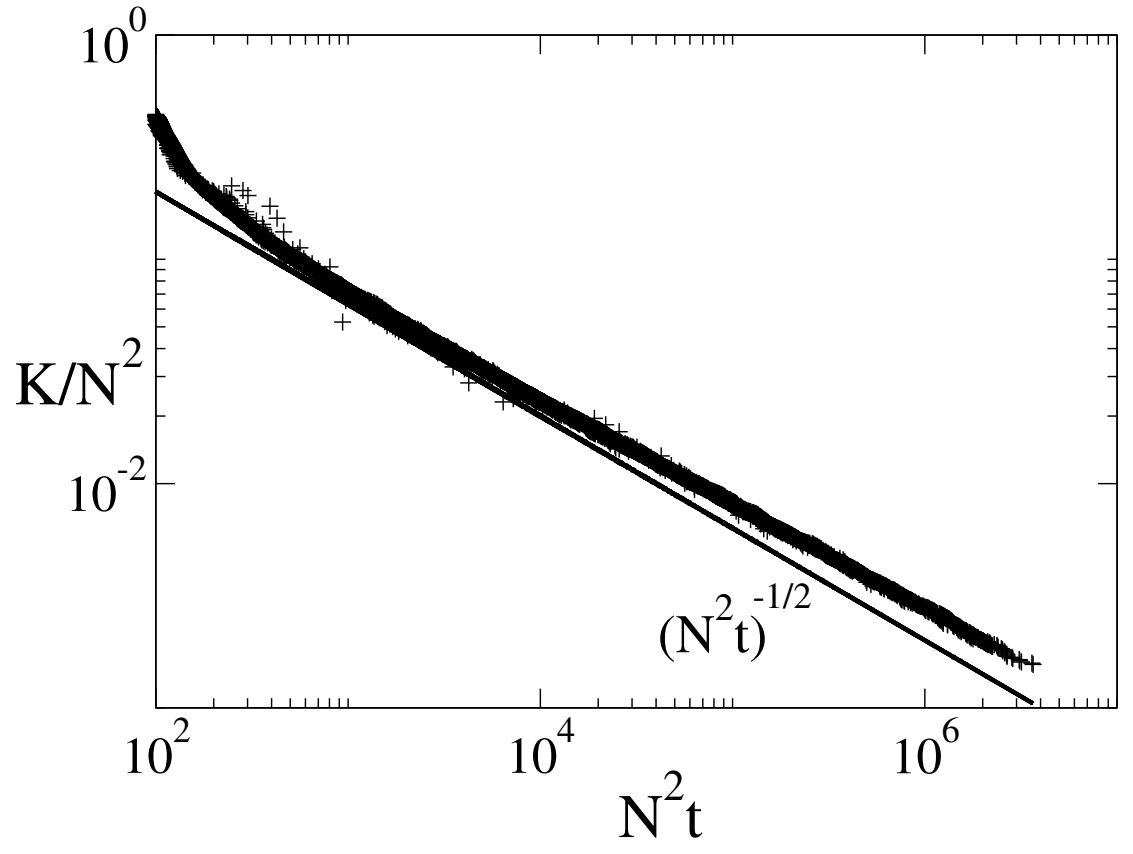

Figure 10: A comparison of the evolving jump density $\frac{K}{N^{2}}$ with the expected coarsening rate (68) for the two-dimensional system (52). 


\section{References}

[1] H. Amann. Time-delayed Perona-Malik type problems. Preprint, 2006.

[2] A. Belahmidi and A. Chambolle. Time-delay regularization of anisotropic diffusion and image processing. M2AN, 39(2):231-251, 2005.

[3] G. Bellettini and G. Fusco. A regularized Perona-Malik functional: some aspects of the gradient dynamics. In H. Broer, J. Mahwin, A. Vanderbauwhede, and S. V. Lunel, editors, EQUADIFF 2003 Proceedings of the International Conference on Differential Equations, Hasselt, Belgium, July 2003.

[4] G. Bellettini and G. Fusco. The gamma limit and the related gradient flow for singular perturbation functionals of Perona-Malik type. Preprint, 2005.

[5] G. Bellettini, M. Novaga, and E. Paolini. Global solutions to the gradient flow equation of a nonconvex functional. To appear in SIAM J. Math. Anal., 2005.

[6] F. Catte, P.-L. Lions, J.-M. Morel, and T. Coll. Image selective smoothing and edge detection by nonlinear diffusion. SIAM J. Numer. Anal., 29(1):182-193, 1992.

[7] Sergio Conti, Barbara Niethammer, and Felix Otto. Coarsening rates in off-critical mixtures. SIAM J. Math. Anal., 37(6):1732-1741 (electronic), 2006.

[8] S. Dai and R. L. Pego. Universal bounds on coarsening rates for mean-field models of phase transitions. SIAM J. Math. Anal., 37(2):347-371 (electronic), 2005.

[9] S. Dai and R. L. Pego. An upper bound on the coarsening rate for mushy zones in a phase-field model. Interfaces Free Bound., 7(2):187-197, 2005.

[10] S. Esedoḡlu. An analysis of the Perona-Malik scheme. Comm. Pure Appl. Math., 54(12):1442-1487, 2001.

[11] M. Ghisi and M. Gobbino. A class of local classical solutions for the one-dimensional Perona-Malik equation. Preprint, 2006.

[12] M. Ghisi and M. Gobbino. Gradient estimates for the Perona-Malik equation. To appear in Math. Ann., 2006.

[13] M. Gobbino. Entire solutions of the one-dimensional Perona-Malik equation. To appear in Comm. Partial Differential Equations, 2003.

[14] D. Horstmann, K. J. Painter, and H. G. Othmer. Aggregation under local reinforcement: from lattice to continuum. European J. Appl. Math., 15(5):546-576, 2004.

[15] B. Kawohl and N. Kutev. Maximum and comparison principle for one-dimensional anisotropic diffusion. Math. Ann., 311:107-123, 1998.

[16] S. Kichenassamy. The Perona-Malik paradox. SIAM J. Appl. Math, 57(5):1328-1342, 1997.

[17] R. V. Kohn and F. Otto. Upper bounds on coarsening rates. Comm. Math. Phys., 229(3):375-395, 2002.

[18] R. V. Kohn and X. Yan. Upper bound on the coarsening rate for an epitaxial growth model. Comm. Pure Appl. Math., 56(11):1549-1564, 2003.

[19] R. V. Kohn and X. Yan. Coarsening rates for models of multicomponent phase separation. Interfaces Free Bound., 6(1):135-149, 2004.

[20] Bo Li and Jian-Guo Liu. Epitaxial growth without slope selection: energetics, coarsening, and dynamic scaling. J. Nonlinear Sci., 14(5):429-451 (2005), 2004.

[21] M. Lizana and V. Padron. A spatially discrete model for aggregating populations. $J$. Math. Biol., 38(1):79-102, 1999.

[22] M. Nitzberg and T. Shiota. Nonlinear image filtering with edge and corner enhancement. IEEE Trans. Pattern Anal. Mach. Intell., 14:826-833, 1992. 
[23] F. Otto, T. Rump, and D. Slepčev. Coarsening rates for a droplet model: Rigorous upper bounds. SIAM J. Math. Anal., 2006. To appear.

[24] K. J. Painter, D. Horstmann, and H. G. Othmer. Localization in lattice and continuum models of reinforced random walks. Appl. Math. Lett., 16(3):375-381, 2003.

[25] P. Perona and J. Malik. Scale space and edge detection using anisotropic diffusion. Technical report, Dept. of EECS Technical Report, U.C. Berkeley, 1987.

[26] P. Perona and J. Malik. Scale-space and edge detection using anisotropic diffusion. IEEE Trans. Pattern. Anal. Machine Intell., 12:629-639, 1990.

[27] W. H. Press, S.A. Teukolsky, W. T. Vetterling, and B. P. Flannery. Numerical recipes in C. Cambridge University Press, Cambridge, second edition, 1992. The art of scientific computing.

[28] M. Shearer, D. G. Schaeffer, and T. P. Witelski. Stability of shear bands in an elastoplastic model for granular flow: the role of discreteness. Math. Models Methods Appl. Sci., 13(11):1629-1671, 2003.

[29] T. P. Witelski, D. G. Schaeffer, and M. Shearer. A discrete model for an ill-posed nonlinear parabolic PDE. Phys. D, 160(3-4):189-221, 2001.

[30] K. Zhang. Existence of infinitely many solutions for the one-dimensional Perona-Malik model. Calc. Var. Partial Differential Equations, 26(2):171-199, 2006. 